\title{
Development and application of proteomics technologies in Saccharomyces cerevisiae
}

\author{
Annemieke Kolkman, Monique Slijper and Albert J.R. Heck
}

Department of Biomolecular Mass Spectrometry, Bijvoet Center for Biomolecular Research and Utrecht Institute for Pharmaceutical Sciences, Utrecht University, Sorbonnelaan 16, 3584CA Utrecht, The Netherlands

\begin{abstract}
Proteomics research focuses on the identification and quantification of 'all' proteins present in cells, organisms or tissue. Proteomics is technically complicated because it encompasses the characterization and functional analysis of all proteins that are expressed by a genome. Moreover, because the expression levels of proteins strongly depend on complex regulatory systems, the proteome is highly dynamic. This review focuses on the two major proteomics methodologies, one based on 2D gel electrophoresis and the other based on liquid chromatography coupled to mass spectrometry. The recent developments of these methodologies and their application to quantitative proteomics are described. The model system Saccharomyces cerevisiae is considered to be the optimal vehicle for proteomics and we review studies investigating yeast adaptation to changes in (nutritional) environment.
\end{abstract}

\section{Yeast as a production platform and model system}

The symbiosis between the yeast Saccharomyces cerevisiae and humans is highly valuable. Evidently, $S$. cerevisiae is one of the most extensively used microorganisms in industrial applications. In addition to its application in the food and beverage industry, S. cerevisiae has been used for the production of protein- and smallmolecule drugs. For example, a substantial part of insulin is produced in $S$. cerevisiae [1]. In addition to its role in industrial applications, $S$. cerevisiae is a key model organism in research laboratories to study fundamental biological processes. Being one of the simplest eukaryotes, $S$. cerevisiae has several features that make it a useful research vehicle [2-4]; it grows easily and methods for cultivation under well-controlled conditions are available. Moreover, $S$. cerevisiae is not pathogenic and, therefore, it can be handled with few precautions. Additionally, powerful techniques for genetic manipulation in yeast are available providing, for example, extensive knockout mutant collections [5-7]. Importantly, many basic biological structures and processes have been conserved throughout eukaryotes and, therefore, S. cerevisiae can be an informative predictor of human gene function. Nearly $50 \%$ of human genes implicated in heritable

\footnotetext{
Corresponding author: Kolkman, A. (a.kolkman@pharm.uu.nl).
}

Available online 3 October 2005 diseases have yeast homologues [8]. Research using yeast has offered generic fundamental insights into processes such as the cell cycle, vesicular transport and gene expression.

Illustrating its importance in molecular biology research, S. cerevisiae was also the first eukaryotic organism from which the genome sequence was completed [9], which has opened up many new possibilities for biotechnological and biomedical research. Yeast has further established its 'super model organism' status [3] as an ideal platform for the development, validation and application of post-genomic technologies, such as those used in large-scale gene knockout genetics and functional genomics studies [2], large-scale analysis of the yeast transcriptome, proteome and metabolome.

\section{Yeast growth conditions}

$S$. cerevisiae can adapt to a large variety of environmental conditions. During fermentation in grapes, for example, yeast encounters sugar concentrations that can vary from $1 \mathrm{M}$ to $10^{-5} \mathrm{M}$ [10]. Also, during baker's yeast production, yeast grows aerobically under sugar limitation to achieve high biomass yields, whereas during processes such as dough rising, high concentrations of fermentable sugars are present under anaerobic conditions and the growth is limited by other nutrients (e.g. oxygen, nitrogen). To survive changes in the nutritional environment, yeast needs to detect the availability of nutrients and adapt its metabolism rapidly. It is important to study how yeast adapts to changes in its environment, not only because it might be relevant for the optimization in industrial applications but also because knowledge gained in such experiments might be relevant to human health and/or malfunctioning.

Comprehensive transcriptome analyses have been performed to study the effect of different nutrient conditions in yeast [11-14]. These genome-wide expression profiles reflect the physiological status of the cell and show how the cell responds to different nutrient environments at the transcription level. Compared with transcriptome studies, proteome studies are generally limited by the number of gene products that can be analyzed simultaneously. However, evidence is accumulating to show that mRNA abundance does not always correlate well with protein expression levels [15-19]. 


\begin{abstract}
Glossary
DiGE: Two-dimensional differential gel-electrophoresis is used to analyze more than one sample on a single $2 \mathrm{D}$ gel. This method employs pre-electrophoretic labelling of proteins with different spectrally resolvable fluorescent cyanide dyes (e.g. Cy2, Сy3, Cy5). The labelled samples are mixed and then separated in a single 2D experiment. The 2D gel patterns of the individual protein pools are visualized by fluorescence excitation, the wavelength of which is unique for each of the different cyanide dyes.

MuDPiT: Multidimensional protein identification technology is used for the direct analysis of complex protein mixtures without 2D gel electrophoresis. In this approach, a complex peptide mixture is loaded on a biphasic column in which two independent stationary phases, namely strong cationic-exchange material and reversed-phase material, are packed into a fused silica nanospray capillary. The chromatography proceeds in cycles; each comprising (i) a step gradient with increasing ionic strength to elute peptides from the strong cationic-exchange material onto the reversed-phase material and (ii) a gradient with increasing concentration of organic modifier to elute peptides from the reversed-phase material into the mass spectrometer. The peptides are eluted directly into the ion source of a mass spectrometer, in which they are fragmented in data-dependent mode.

ICAT: Isotope-coded affinity tag technology was developed to selectively label cysteine residues in proteins with tags that differ in their isotopic content, enabling relative quantification of peptide abundance between samples. The ICAT reagent consists of three parts: (i) a biotin moiety, which is used for selective isolation of the labelled peptides, (ii) an isotopically labelled linker, which contains either the heavy or the light isotopes and (iii) a reactive group that specifically alkylate thiol groups in cysteine residues.

iTRAQ: A multiplexed set of chemical reagents for quantitative proteome analysis that enables relative quantification on the basis of MS-MS spectra. These so-called ITRAO reagents are amine-specific and covalently attach isobaric mass labels to the $\mathrm{N}$-terminus and to lysine side chains of peptides. They can be used to label all the peptides in up to four different biological samples, simultaneously: enabling relative quantification from MS-MS spectra. The derivatized precursor peptides are identical in mass and thus indistinguishable in MS spectra but produce strong diagnostic MS-MS signature ions.
\end{abstract}

Therefore, it is essential to study yeast adaptation and other biological processes at the level of the proteome.

Here, we review established and emerging (quantitative) proteomics methodologies that often have first been developed and validated in S. cerevisiae. The review mainly focuses on two major proteomics methodologies available nowadays, namely $2 \mathrm{D}$ gel electrophoresis and liquid chromatography coupled to mass spectrometry (LC-MS). Additionally, we focus on how these technologies can be used to investigate differential protein expression levels of yeast grown under different growth conditions as used in industrial applications. We conclude with a brief discussion on future prospects for proteomics technologies and the role of proteomics and $S$. cerevisiae in the emerging field of systems biology.

\section{Coverage of the yeast proteome}

\section{$2 D$-gel-based proteomics}

The conventional workflow in proteomics research employs 2D gel electrophoresis to separate proteins and mass spectrometry (MS) to identify proteins. On a 2D gel, proteins are separated using iso-electric focusing in the first dimension and sodium dodecyl sulfate polyacrylamide gel electrophoresis in the second dimension (Figure 1a,b). Separated proteins can be visualized using a variety of post-electrophoretic staining methods, such as colloidal Coomassie blue dyes, silver stains and fluorescent dyes (for reviews, see [20,21]). Although 2D gel electrophoresis was introduced 30 years ago, its application to proteomics has really taken off since the development of MS-based techniques that enabled high-throughput protein identification (for reviews, see [22-24]).

The application of $2 \mathrm{D}$ gel electrophoresis to $S$. cerevisiae for the large-scale separation and visualization of proteins results in so-called yeast reference maps [2529]. Depending on the protein staining method, $\sim 1000$ proteins can be visualized on such gels. Also, subproteome reference maps of, for example, yeast mitochondria, have been generated [30]. Moreover, 2D reference maps have been constructed for important industrial yeast strains, such as an ale-fermenting strain [31], a wine strain [32] and a lager-brewing strain [33,34]. These annotated reference maps are useful tools for yeast researchers because they can be used for 2D gel comparisons; however, because of poor gel-to-gel reproducibility and strain variation, protein spot identities should always be confirmed using MS.

\section{LC-based proteomics}

Although 2D gel electrophoresis enables the separation of thousands of proteins simultaneously and is still one of the most frequently used methods in proteome analysis, it has its drawbacks: the technique is labour-intensive and is hampered by poor gel-to-gel reproducibility; low abundant proteins and hydrophobic proteins, in particular membrane proteins, are generally not observed; and the gels have a limited dynamic range of detection $\left(1-10^{4}\right)$ [35]. In recent years, proteomics methods that employ liquid chromatography (LC) coupled to MS have proven to provide strong alternatives (for reviews, see [36,37]). LCbased technologies have several advantages compared with 2D-gel-based techniques. LC-MS, which can be automated, combines high-speed, high-resolution and high-sensitivity separation of extremely complex peptide mixtures.

An example of an innovative on-line 2D chromatographic approach is the 'multidimensional protein identification technology' (MudPIT; see Glossary) [38,39]. This approach starts with the proteolysis of a complete cellular protein mixture. The resulting peptide mixture is then separated and analyzed using on-line 2D chromatography directly coupled to tandem MS, which enables the identification of proteins by peptide sequencing. In one of the first applications, Washburn et al. applied MudPIT to analyze the proteome of yeast and a total of 1484 could be identified [40]. The resulting dataset was relatively unprejudiced, covering proteins from all subcellular compartments, with wide-ranging isoelectric points and molecular weights. Moreover, low abundance proteins, such as transcription factors and protein kinases, as well as hydrophobic membrane proteins, were detected. More recently, Wei et al. [41] improved the MudPIT method by adding an additional reversed phase column to the biphasic column, resulting in an on-line 3D LC method. Wei et al. identified 3109 yeast proteins, which is possibly the most comprehensive proteome coverage reported to date.

\section{Quantification strategies}

\section{D-gel-based quantification}

It is interesting to know which proteins are present in a cell. However, it is even more important to know 
(a)

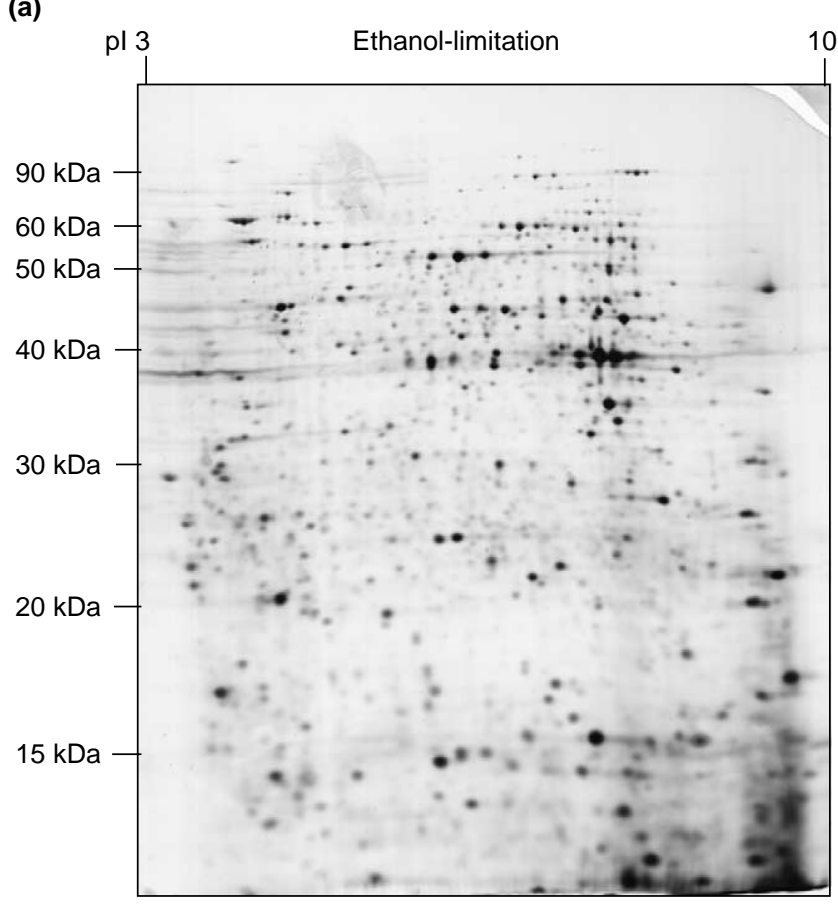

(c)

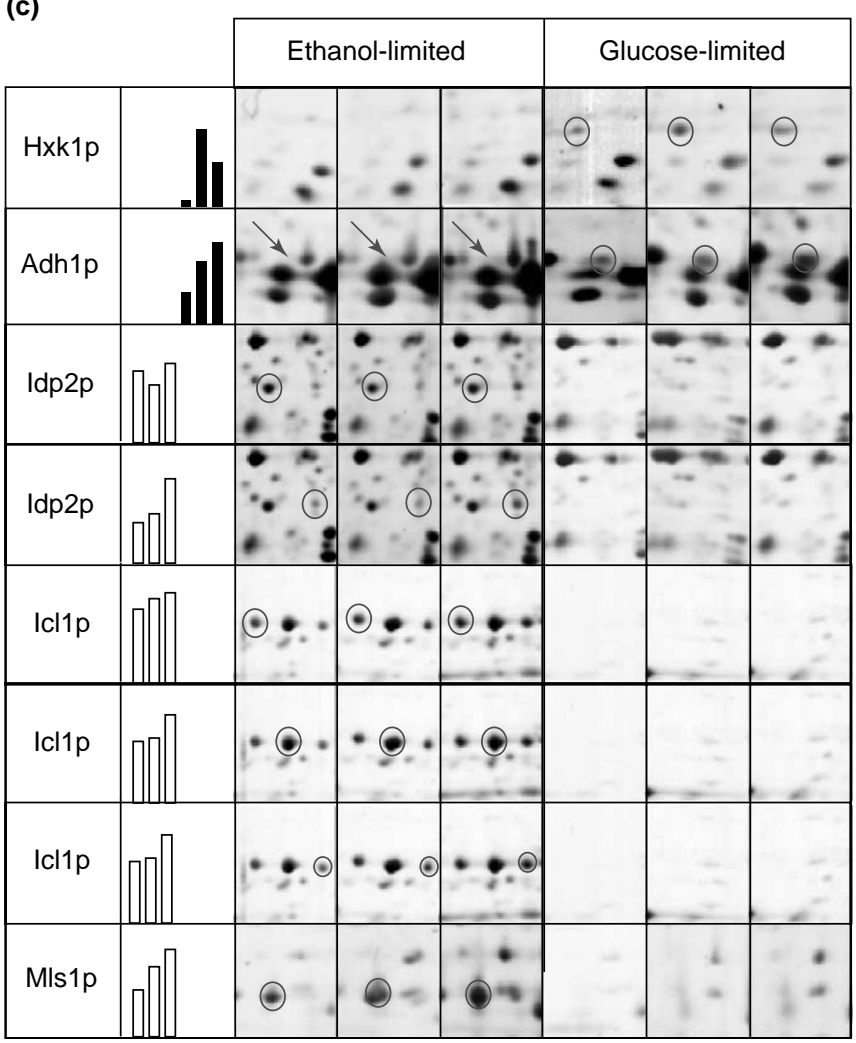

(b)

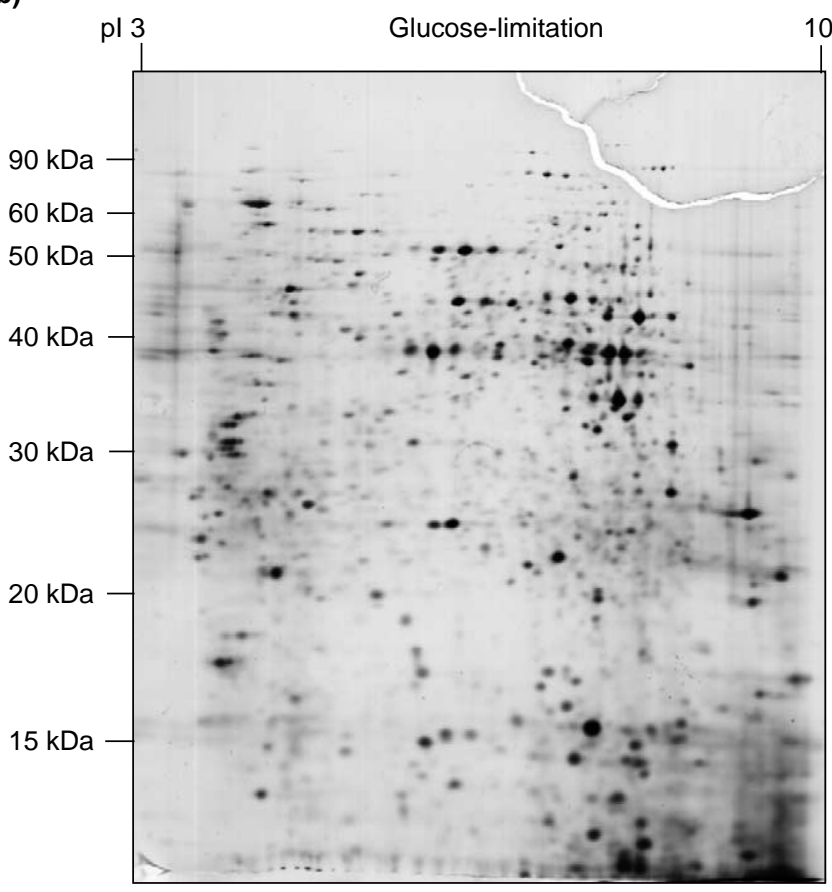

(d)

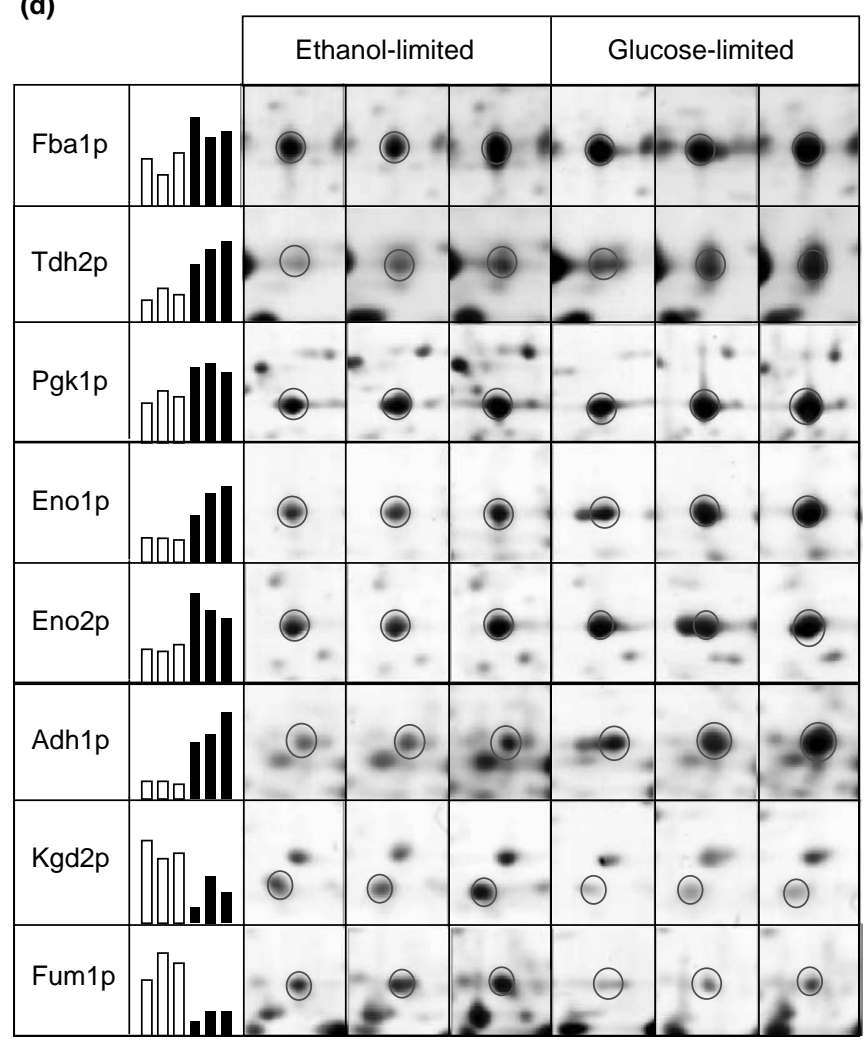

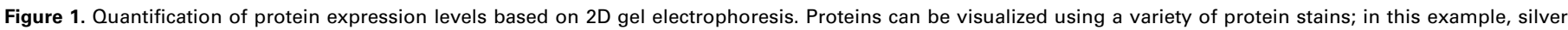
staining was used. The images are of $2 \mathrm{D}$ gels loaded with $150 \mu \mathrm{g}$ protein extract from yeast cells, which were grown in aerobic chemostat cultures: (a) carbon-limited for

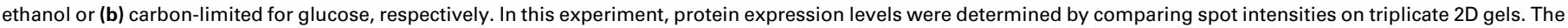

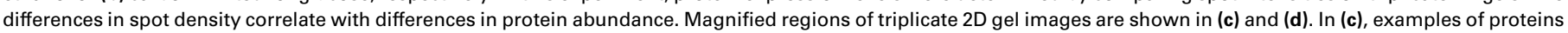

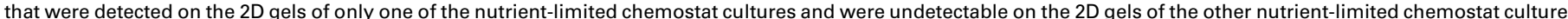

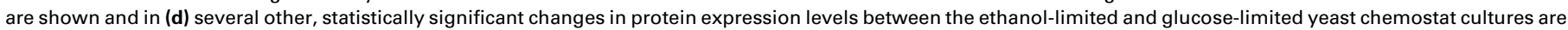

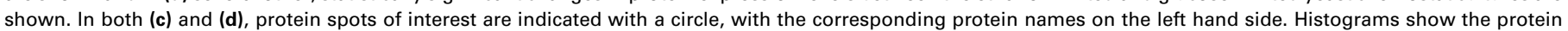

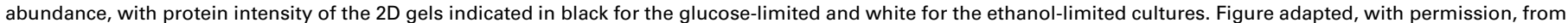
Ref. [44]. 
the (relative) abundance of these proteins. Conventionally, determination of protein expression levels has been accomplished by comparing spot intensities from $2 \mathrm{D}$ gels (Figure 1c,d). In this way, changes in individual protein spots can be detected and quantified. Several commercially available software packages have been developed to perform the image analysis of $2 \mathrm{D}$ gels (for a review, see [42]).

Many quantitative 2D-gel-based studies have been reported with applications to yeast, examining, for example, yeast growth under different environmental conditions. Salusjarvi et al. [43] performed a proteome analysis of recombinant xylose-fermenting yeast, comparing conditions in which glucose or xylose was the carbon source. This study revealed that metabolic fluxes in the acetate and glycerol pathway were significantly different in cells growing on xylose compared with those growing on glucose. Kolkman et al. studied yeast grown in chemostat cultures limited for glucose and ethanol [44], which enabled the differential analysis of protein expression levels under glycolytic and gluconeogenic conditions. By using multiple 2D gels (Figure 1c,d) it was possible to perform a decent statistical analysis to determine significant changes in protein expression levels. This study revealed exclusively major changes in the central carbon metabolism pathways upon changing the carbon source. In other studies, $2 \mathrm{D}$ gel electrophoresis was used to obtain a global view of changes in the yeast proteome as a function of stimuli in the environment, such as cadmium [45], lithium [46], $\mathrm{H}_{2} \mathrm{O}_{2}$ [47], sorbic acid [48] and amino acid starvation [49].

There are a few examples in which more physiological relevant conditions, such as wine making, baking and brewing, were used to study changes in the yeast proteome. Brejning et al. [50] identified proteins whose expression was induced during the lag phase and growth initiation in lager brewing yeast. Trabalzini et al. [51] performed a proteome analysis to study the adaptive response of a wine yeast strain to mild and progressive physiological stresses that occur during fermentation. Good wine-yeast-strains should be able to adapt to such stress. Proteome knowledge about these industrial strains can, therefore, be useful for the optimization and control of yeast proliferation during industrial fermentation.

\section{Difference in gel electrophoresis}

To overcome some of the intrinsic disadvantages of $2 \mathrm{D}$ gel electrophoresis, such as gel-to-gel variation and poor reproducibility, Unlu et al. [52,53] developed an approach, termed 'difference in gel electrophoresis' (DiGE; see Glossary) (Figure 2). This method employs pre-electrophoretic labelling of proteins with up to three different fluorescent cyanide dyes, which allows the use of an internal standard in each gel to reduce the effects of gel-togel variation [54]. A comparison of the resulting images enables quantification based on the total fluorescence

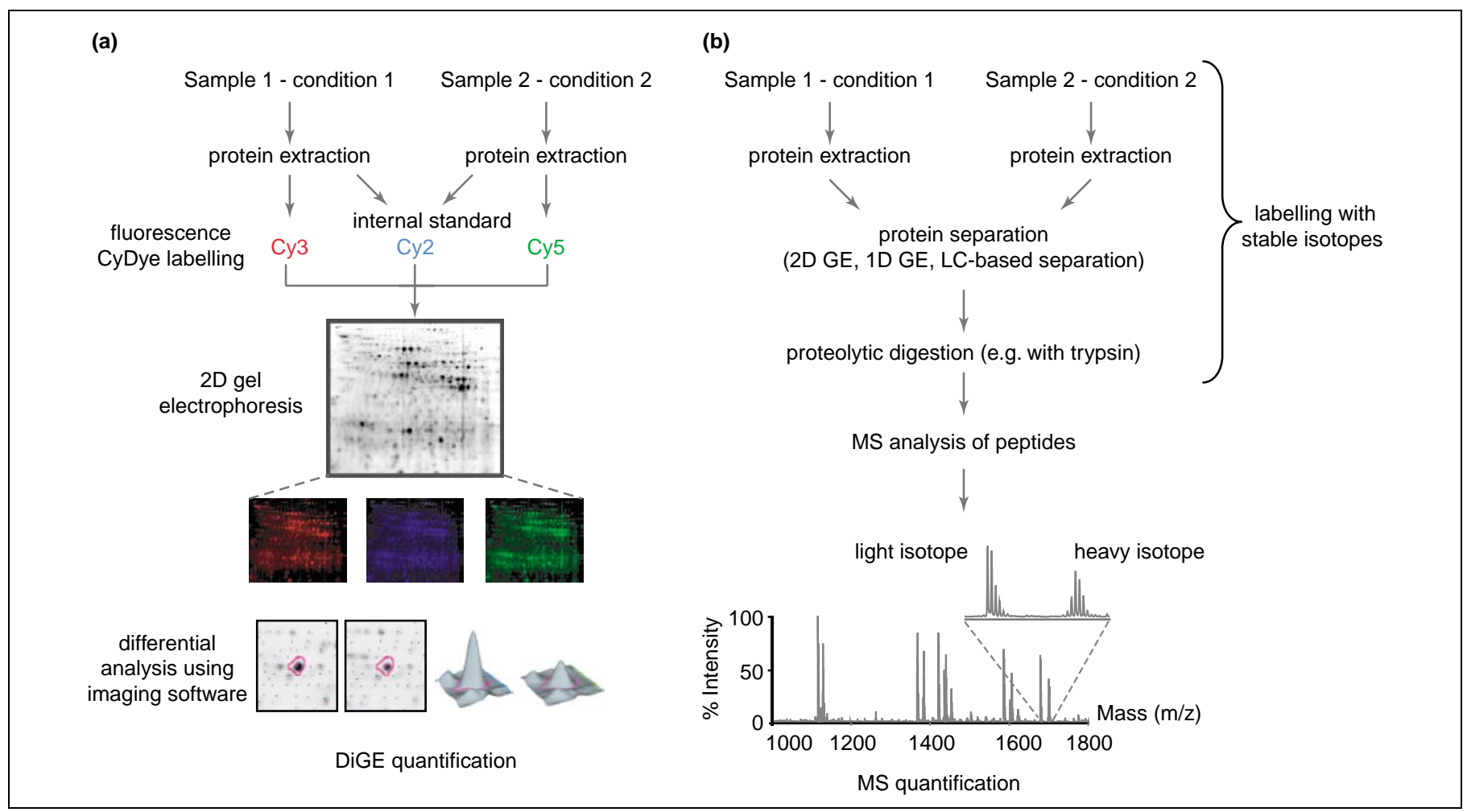

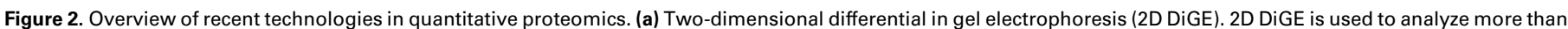

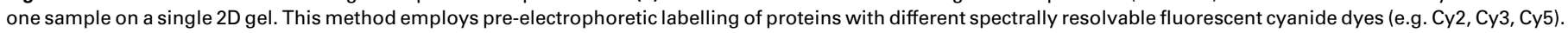

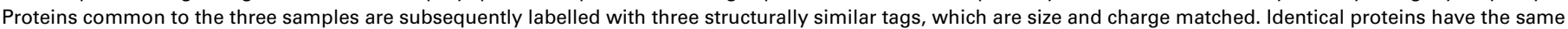

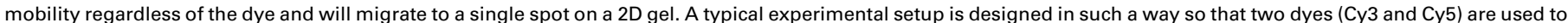

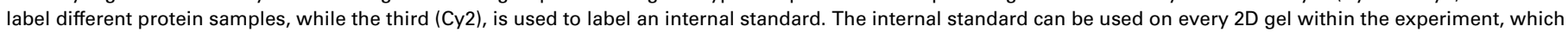

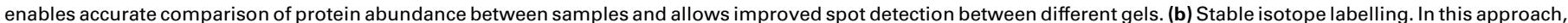

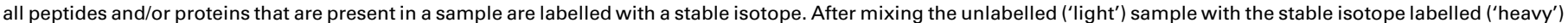
sample, the sample is processed and analyzed by mass spectrometry to determine relative protein expression levels. Figure adapted, with permission, from Ref. [63]. 


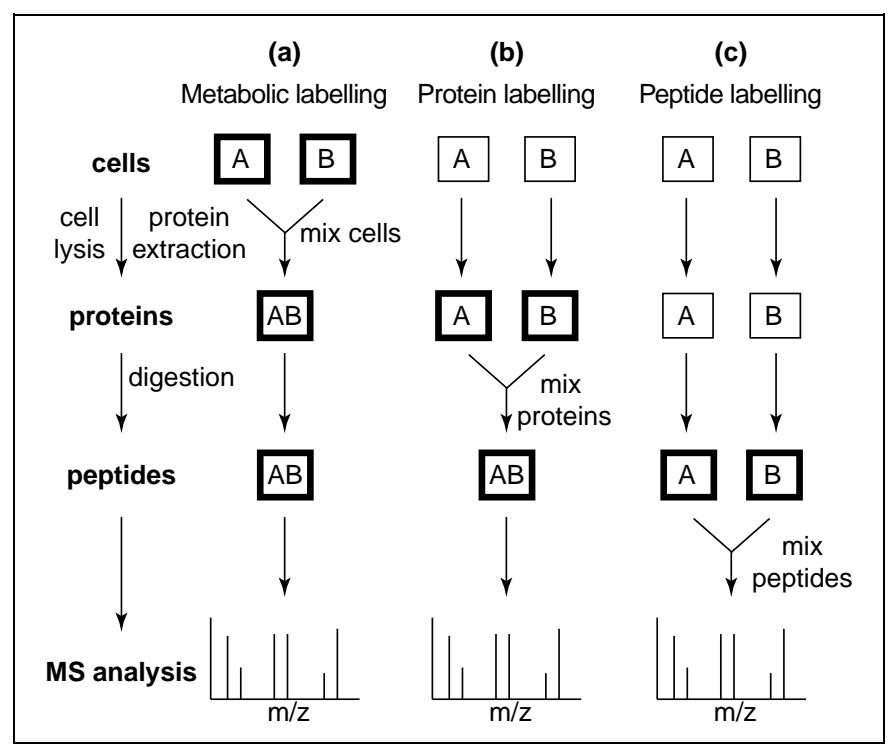

Figure 3. An overview of different strategies for quantification through stableisotope labelling and mass spectrometry. These protein quantification methods make use of stable isotope labels (i.e. chemically identical but mass-differentiated tags) to label all peptides and/or proteins that are present in a sample. After mixing the unlabelled sample with the stable-isotope-labelled sample, the sample is processed and analyzed by mass spectrometry to determine relative protein expression levels. The mass spectrometric intensity response is independent of the isotopic composition of the peptides. The ratio of the integrated signal peak areas of the light and heavy isotopic form of the same peptide, therefore directly reflect the protein abundance ratio. The stable isotope label can be incorporated into proteins or peptides at different moments during sample preparation (labelling is indicated with black bold boxes). The methods are: (a) Metabolic labelling. Metabolic labelling is achieved by growing an organism in the presence of a stable- isotopelabelled nitrogen or carbon source, such as ${ }^{15} \mathrm{~N}$ ammonium sulphate, ${ }^{13} \mathrm{C}$-labelled glucose or labelled amino acids. In a typical labelling study, cells are grown under different conditions and labelled with different forms of the stable isotope. Subsequently, these cells are mixed and analyzed using mass spectrometry; (b) Protein labelling (e.g. the ICAT approach); and (c) Peptide labelling, either during digestion in ${ }^{18} \mathrm{O}$ labelled water, via derivatization of the peptides or via methods such as ITRAQ.

intensity of each protein spot. Hu et al. [55] used DiGE to compare the proteome of yeast upon treatment with 15 metals. Their analysis revealed that $\sim 20 \%$ of the proteins showed a significant change in expression upon metal treatment, providing insight into cellular defense mechanisms in eukaryotes. Proteins such as $\mathrm{Cu} / \mathrm{Zn}$ superoxide dismutase showed drastic changes in expression upon treatment with different metals, which is probably a result of their overall role in the detoxification of metals. Interestingly, certain proteins only changed their expression levels upon treatment with a specific metal, indicating that these proteins might have a specific role in the cellular defence.

\section{Stable-isotope-labelling-based quantification}

Recently, MS-based strategies for quantitative proteomics have been developed. These methods make use of stable isotope labels, that is, chemically identical but massdifferentiated tags to label peptides and/or proteins (Figures 2 and 3; for reviews, see [56-58]). Three main methods exist for introducing stable isotopes into proteins or peptides: chemical, enzymatic or via metabolic incorporation.

Various groups have applied stable-isotope labelling in yeast to study the effect of different environmental conditions on the proteome. For example, Ideker et al. [18] and Griffin et al. [19] applied the chemical incorporation method ICAT (see Glossary) [59] to study the effect of carbon source perturbation on protein expression levels in yeast. In both studies, the protein abundance ratios were compared with corresponding mRNA-abundance ratios. Ross et al. applied the iTRAQ method (see Glossary) to determine global protein expression levels in a wild type yeast strain and in two mutant yeast strains that are defective in the nonsense mRNA decay and general $5^{\prime}$ to $3^{\prime}$ decay [60].

Metabolic labelling has been applied to yeast in various studies to determine relative protein expression levels. In fact, for proteome studies, yeast was one of the first organisms to be fully metabolically labelled with stable isotopes for the purpose of quantitative MS measurements [61]. Metabolic labelling offers the earliest time point for stable-isotope incorporation and, therefore, is less prone to experimental variations that might occur during parallel sample processing. However, a limitation of this approach is that the organism to be labelled needs to grow in controlled media and thus metabolic labelling is mainly limited to organisms that can be cultured and to cell lines.

Washburn et al. [62] used metabolic labelling in yeast with ${ }^{15} \mathrm{~N}$ as the stable isotope, in combination with quantitative MudPIT analysis. They compared the

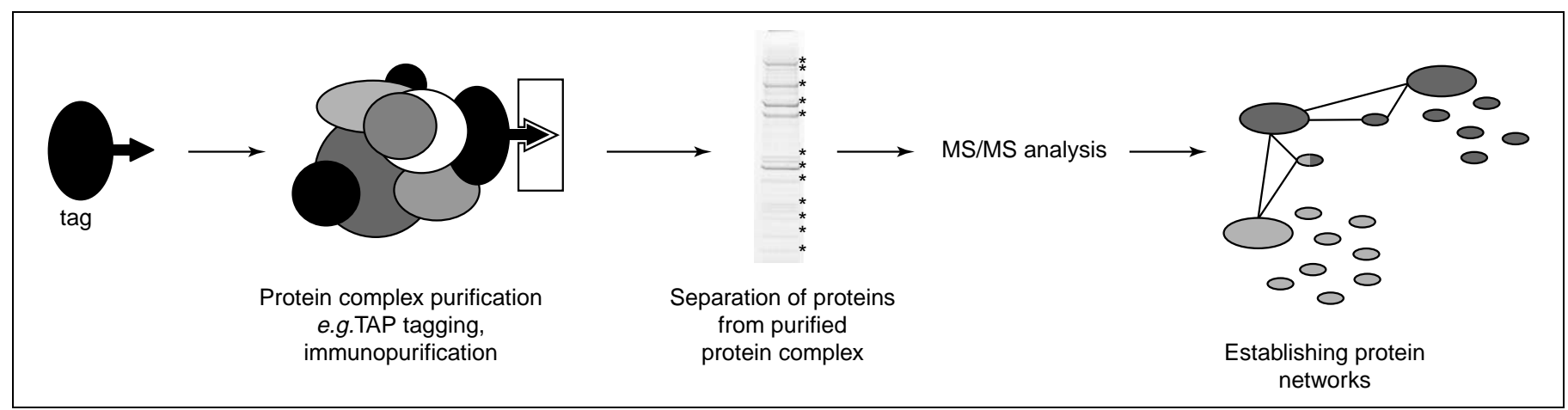

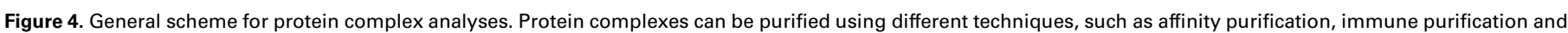

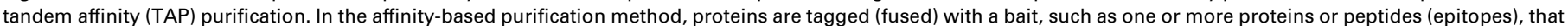

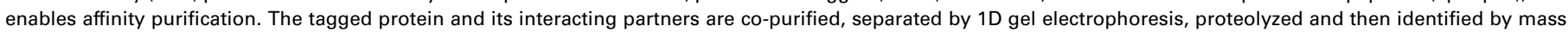

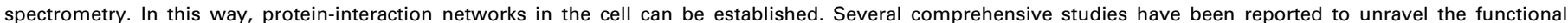

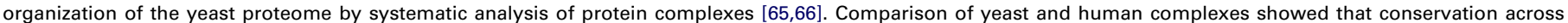

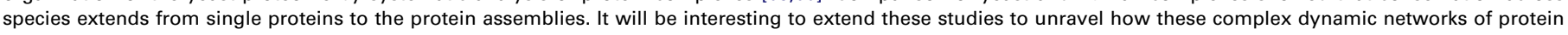
interactions adapt to biological processes, such as cell cycle and budding, and also to changes in environmental growth conditions. 
proteome and transcriptome of yeast cultured on minimal and rich media, in which ${ }^{14} \mathrm{~N}$ or ${ }^{15} \mathrm{~N}$ labelled ammonium sulfate was present as the sole nitrogen source. A clustering approach based on protein biochemical pathways and protein complexes was applied, which provided insight into generic yeast biology.

\section{Comparison of technologies for quantitative proteomics} Quantitative protein expression profiling is a crucial part of proteomics and requires methods that can efficiently provide accurate and reproducible differential expression values for proteins in two or more biological samples. It is clear from earlier that 'older' staining methods combined with $2 \mathrm{D}$ gel electrophoresis have some drawbacks, largely resulting from experimental variation in gel-to-gel reproducibility. Therefore, both stable isotope labelling and DiGE currently appear to be the methods of choice for reliable protein expression quantification. In the stable isotope approach, the reliability of the quantification increases with the number of peptides of the same protein that are used for quantification. Recently, in a direct comparative assessment, Kolkman et al. [63] evaluated DiGE and metabolic stable-isotope labelling. Relative ratios in protein expression between these two yeast samples were determined using both DiGE and metabolic stable-isotope labelling and a satisfactory correlation between the two methods was found, although each method has their intrinsic advantages and limitations [63].

\section{Future outlook - towards systems biology}

Proteomics is a rapidly developing area of research, whereby new technologies are often developed and validated in model systems such as yeast. However, compared with genetics and genomics studies, proteomics is still limited because it is strongly biased towards highly abundant proteins and, therefore, does not yet provide the genome-wide coverage obtained by other -omics technologies. Additionally, the proteome world is possibly the most complex of all -omics worlds because of its highly dynamic nature and its complexity resulting from splicevariants, isoforms and protein post-translational modifications. Therefore, it is evident that there is an ongoing need for improvement in (quantitative) proteomics technologies, whereby yeast will probably have its role again as the benchmark model system.

Instead of proteins acting as individual entities, most proteins interact and/or function in large molecular assemblies. It is now assumed that the diversity of biological processes is derived from the dynamic network of protein interactions in the cell [64]. Thus, a broad understanding of protein-protein interactions is crucial for building models of protein function and regulation. With its speed and sensitivity, MS has become an important method for identifying components of protein complexes (Figure 4). Several comprehensive studies have been reported to unravel the functional organization of the yeast proteome by systematic analysis of protein complexes in yeast [65,66]. Quantitative proteomics techniques using stable isotopes can be used to study these protein complexes; for example, to distinguish specific from aspecific interactions, and to determine protein complex dynamics and the stoichiometry of protein complexes [67].

The combination of genetics and a wide variety of omics data (transcriptomics, proteomics, metabolomics), can potentially lead to a more complete understanding of the biology of a system at the molecular level. This ambitious quest is nowadays termed systems biology (for review, see [68]). However, for such systems biology experiments the quality and range (in time and coverage) of the different -omics data should be comparable. It is crucial to make the systems studied as 'simple' as possible, therefore, it is clear that the development of systems biology will benefit largely from model systems that can be grown and manipulated under well-defined conditions, providing again a role for $S$. cerevisiae at the centre stage.

\section{Acknowledgements}

This work was supported by the Netherlands Proteomics Centre (http:// www.netherlandsproteomicscentre.nl).

\section{References}

1 Kjeldsen, T. (2000) Yeast secretory expression of insulin precursors. Appl. Microbiol. Biotechnol. 54, 277-286

2 Kumar, A. and Snyder, M. (2001) Emerging technologies in yeast genomics. Nat. Rev. Genet. 2, 302-312

3 Barr, M.M. (2003) Super models. Physiol. Genomics 13, 15-24

4 Castrillo, J.I. and Oliver, S.G. (2004) Yeast as a touchstone in postgenomic research: strategies for integrative analysis in functional genomics. J. Biochem. Mol. Biol. 37, 93-106

5 Mnaimneh, S. et al. (2004) Exploration of essential gene functions via titratable promoter alleles. Cell 118, 31-44

6 Winzeler, E.A. et al. (1999) Functional characterization of the $S$. cerevisiae genome by gene deletion and parallel analysis. Science 285 , 901-906

7 Giaever, G. et al. (2002) Functional profiling of the Saccharomyces cerevisiae genome. Nature 418, 387-391

8 Bassett, D.E., Jr. et al. (1996) Yeast genes and human disease. Nature 379, 589-590

9 Goffeau, A. et al. (1996) Life with 6000 genes. Science 274, 563-567

10 Kruckeberg, A.L. (1996) The hexose transporter family of Saccharomyces cerevisiae. Arch. Microbiol. 166, 283-292

11 Boer, V.M. et al. (2003) The genome-wide transcriptional responses of Saccharomyces cerevisiae grown on glucose in aerobic chemostat cultures limited for carbon, nitrogen, phosphorus or sulfur. J. Biol. Chem. 278, 3265-3274

$12 \mathrm{Wu}$, J. et al. (2004) Global analysis of nutrient control of gene expression in Saccharomyces cerevisiae during growth and starvation. Proc. Natl. Acad. Sci. U. S. A. 101, 3148-3153

13 Saldanha, A.J. et al. (2004) Nutritional homeostasis in batch and steady-state culture of yeast. Mol. Biol. Cell 15, 4089-4104

14 Tai, S.L. et al. (2005) Two-dimensional transcriptome analysis in chemostat cultures. Combinatorial effects of oxygen availability and macronutrient limitation in Saccharomyces cerevisiae. J. Biol. Chem. $280,437-447$

15 Anderson, L. and Seilhamer, J. (1997) A comparison of selected mRNA and protein abundances in human liver. Electrophoresis 18, 533-537

16 Gygi, S.P. et al. (1999) Correlation between protein and mRNA abundance in yeast. Mol. Cell. Biol. 19, 1720-1730

17 Le Naour, F. et al. (2001) Profiling changes in gene expression during differentiation and maturation of monocyte-derived dendritic cells using both oligonucleotide microarrays and proteomics. J. Biol. Chem. 276, 17920-17931

18 Ideker, T. et al. (2001) Integrated genomic and proteomic analyses of a systematically perturbed metabolic network. Science 292, 929-934

19 Griffin, T.J. et al. (2002) Complementary profiling of gene expression at the transcriptome and proteome levels in Saccharomyces cerevisiae. Mol. Cell. Proteomics 1, 323-333

20 Rabilloud, T. (2000) Detecting proteins separated by 2-D gel electrophoresis. Anal. Chem. 72, 48A-55A 
21 Patton, W.F. (2002) Detection technologies in proteome analysis. J. Chromatogr. B Analyt. Technol. Biomed. Life Sci. 771, 3-31

22 Mann, M. et al. (2001) Analysis of proteins and proteomes by mass spectrometry. Annu. Rev. Biochem. 70, 437-473

23 Steen, H. and Mann, M. (2004) The ABC's (and XYZ's) of peptide sequencing. Nat. Rev. Mol. Cell Biol. 5, 699-711

24 Yates, J.R., 3rd. (2004) Mass spectral analysis in proteomics. Annu. Rev. Biophys. Biomol. Struct. 33, 297-316

25 Shevchenko, A. et al. (1996) Linking genome and proteome by mass spectrometry: large-scale identification of yeast proteins from two dimensional gels. Proc. Natl. Acad. Sci. U. S. A. 93, 14440-14445

26 Maillet, I. et al. (1996) Rapid identification of yeast proteins on twodimensional gels. J. Biol. Chem. 271, 10263-10270

27 Norbeck, J. and Blomberg, A. (1997) Two-dimensional electrophoretic separation of yeast proteins using a non-linear wide range ( $\mathrm{pH} \mathrm{3-10)}$ immobilized $\mathrm{pH}$ gradient in the first dimension; reproducibility and evidence for isoelectric focusing of alkaline ( $\mathrm{pI}>7$ ) proteins. Yeast 13, 1519-1534

28 Perrot, M. et al. (1999) Two-dimensional gel protein database of Saccharomyces cerevisiae (update 1999). Electrophoresis 20, 2280-2298

29 Wildgruber, R. et al. (2002) Web-based two-dimensional database of Saccharomyces cerevisiae proteins using immobilized $\mathrm{pH}$ gradients from $\mathrm{pH} 6$ to $\mathrm{pH} 12$ and matrix-assisted laser desorption/ionizationtime of flight mass spectrometry. Proteomics 2, 727-732

30 Ohlmeier, S. et al. (2004) The yeast mitochondrial proteome, a study of fermentative and respiratory growth. J. Biol. Chem. 279, 3956-3979

31 Kobi, D. et al. (2004) Two-dimensional protein map of an "ale"-brewing yeast strain: proteome dynamics during fermentation. FEMS Yeast Res 5, 213-230

32 Trabalzini, L. et al. (2003) Proteomic characterization of a wild-type wine strain of Saccharomyces cerevisiae. Ital. J. Biochem. 52, 145-153

33 Joubert, R. et al. (2000) Two-dimensional gel analysis of the proteome of lager brewing yeasts. Yeast 16, 511-522

34 Joubert, R. et al. (2001) Identification by mass spectrometry of twodimensional gel electrophoresis-separated proteins extracted from lager brewing yeast. Electrophoresis 22, 2969-2982

35 Fey, S.J. and Larsen, P.M. (2001) 2D or not 2D. Two-dimensional gel electrophoresis. Curr. Opin. Chem. Biol. 5, 26-33

36 Romijn, E.P. et al. (2003) Recent liquid chromatographic-(tandem) mass spectrometric applications in proteomics. J. Chromatogr. A. $1000,589-608$

37 Shi, Y. et al. (2004) The role of liquid chromatography in proteomics. J. Chromatogr. A. 1053, 27-36

38 Link, A.J.et al. (1999) Direct analysis of protein complexes using mass spectrometry. Nat. Biotechnol. 17, 676-682

39 Wolters, D.A. et al. (2001) An automated multidimensional protein identification technology for shotgun proteomics. Anal. Chem. 73, $5683-5690$

40 Washburn, M.P. et al. (2001) Large-scale analysis of the yeast proteome by multidimensional protein identification technology. Nat. Biotechnol. 19, 242-247

41 Wei, J. et al. (2005) Global proteome discovery using an online threedimensional LC-MS/MS. J. Proteome Res. 4, 801-808

42 Dowsey, A.W. et al. (2003) The role of bioinformatics in twodimensional gel electrophoresis. Proteomics 3, 1567-1596

43 Salusjarvi, L. et al. (2003) Proteome analysis of recombinant xylosefermenting Saccharomyces cerevisiae. Yeast 20, 295-314

44 Kolkman, A. et al. (2005) Comparative proteome analysis of Saccharomyces cerevisiae grown in chemostat cultures limited for glucose or ethanol. Mol. Cell. Proteomics 4, 1-11

45 Vido, K. et al. (2001) A proteome analysis of the cadmium response in Saccharomyces cerevisiae. J. Biol. Chem. 276, 8469-8474
46 Bro, C. et al. (2003) Transcriptional, proteomic, and metabolic responses to lithium in galactose-grown yeast cells. J. Biol. Chem. $278,32141-32149$

47 Godon, C. et al. (1998) The $\mathrm{H}_{2} \mathrm{O}_{2}$ stimulon in Saccharomyces cerevisiae. J. Biol. Chem. 273, 22480-22489

48 de Nobel, H. et al. (2001) Parallel and comparative analysis of the proteome and transcriptome of sorbic acid-stressed Saccharomyces cerevisiae. Yeast 18, 1413-1428

49 Yin, Z. et al. (2004) Proteomic response to amino acid starvation in Candida albicans and Saccharomyces cerevisiae. Proteomics 4, 24252436

50 Brejning, J. et al. (2005) Identification of genes and proteins induced during the lag and early exponential phase of lager brewing yeasts. J. Appl. Microbiol. 98, 261-271

51 Trabalzini, L. et al. (2003) Proteomic response to physiological fermentation stresses in a wild-type wine strain of Saccharomyces cerevisiae. Biochem. J. 370, 35-46

52 Unlu, M. et al. (1997) Difference gel electrophoresis: a single gel method for detecting changes in protein extracts. Electrophoresis 18, 2071-2077

53 Unlu, M. (1999) Difference gel electrophoresis. Biochem. Soc. Trans. $27,547-549$

54 Alban, A. et al. (2003) A novel experimental design for comparative twodimensional gel analysis: two-dimensional difference gel electrophoresis incorporating a pooled internal standard. Proteomics 3, 36-44

$55 \mathrm{Hu}$, Y. et al. (2003) Proteome analysis of Saccharomyces cerevisiae under metal stress by two-dimensional differential gel electrophoresis. Electrophoresis 24, 1458-1470

56 Julka, S. and Regnier, F. (2004) Quantification in proteomics through stable isotope coding: a review. J. Proteome Res. 3, 350-363

57 Ong, S.E. et al. (2003) Mass spectrometric-based approaches in quantitative proteomics. Methods 29, 124-130

58 Sechi, S. and Oda, Y. (2003) Quantitative proteomics using mass spectrometry. Curr. Opin. Chem. Biol. 7, 70-77

59 Gygi, S.P. et al. (1999) Quantitative analysis of complex protein mixtures using isotope-coded affinity tags. Nat. Biotechnol. 17, 994-999

60 Ross, P.L. et al. (2004) Multiplexed protein quantitation in Saccharomyces cerevisiae using amine-reactive isobaric tagging reagents. Mol. Cell. Proteomics 3, 1154-1169

61 Oda, Y. et al. (1999) Accurate quantitation of protein expression and site-specific phosphorylation. Proc. Natl. Acad. Sci. U. S. A. 96, 65916596

62 Washburn, M.P. et al. (2003) Protein pathway and complex clustering of correlated mRNA and protein expression analyses in Saccharomyces cerevisiae. Proc. Natl. Acad. Sci. U. S. A. 100, 3107-3112

63 Kolkman, A. et al. (2005) Double standards in quantitative proteomics: direct comparative assessment of difference in gel electrophoresis and metabolic stable isotope labeling. Mol. Cell. Proteomics 4, 255-266

64 Alberts, B. (1998) The cell as a collection of protein machines: preparing the next generation of molecular biologists. Cell 92, 291-294

65 Gavin, A.C. et al. (2002) Functional organization of the yeast proteome by systematic analysis of protein complexes. Nature $415,141-147$

66 Ho, Y. et al. (2002) Systematic identification of protein complexes in Saccharomyces cerevisiae by mass spectrometry. Nature $415,180-183$

67 Ranish, J.A. et al. (2003) The study of macromolecular complexes by quantitative proteomics. Nat. Genet. 33, 349-355

68 Hohmann, S. (2005) The Yeast Systems Biology Network: mating communities. Curr. Opin. Biotechnol. 16, 356-360

\section{Free journals for developing countries}

The WHO and six medical journal publishers have launched the Access to Research Initiative, which enables nearly 70 of the world's poorest countries to gain free access to biomedical literature through the Internet.

Gro Harlem Brundtland, director-general for the WHO, said that this initiative was 'perhaps the biggest step ever taken towards reducing the health information gap between rich and poor countries'.

See http://www.healthinternetwork.net for more information. 